\title{
ON TRIPLE CODERIVATIONS OF CORINGS
}

\author{
Hiroaki Komatsu and Atsushi Nakajima \\ Received: 12 April 2014; Revised: 19 August 2014 \\ Communicated by Abdullah Harmancı
}

\begin{abstract}
We introduce the notion of a triple coderivation, which is a triplet of maps from a $(\mathcal{C}, \mathcal{C})$-bicomodule to a coring $\mathcal{C}$ satisfying a certain condition closely related to the definition of a coderivation. We determine the structure of the module consisting of all triple coderivations.
\end{abstract}

Mathematics Subject Classification (2010): 16T15

Keywords: Coring, triple coderivation, symmetric generalized coderivation

\section{Introduction}

Throughout this note, $A$ represents an associative algebra with a unit over a commutative ring $R$ and $\mathcal{C}$ an $A$-coring with the coproduct $\Delta$ and with the counit $\varepsilon$. Notations are based on [2]. The category of unitary $(A, A)$-bimodules whose left and right actions of $R$ coincide is denoted by ${ }_{A} \mathbf{M}_{A}$, and the category of counitary $(\mathcal{C}, \mathcal{C})$-bicomodules is denoted by ${ }^{\mathcal{C}} \mathbf{M}^{\mathcal{C}}$. For $M \in{ }^{\mathcal{C}} \mathbf{M}^{\mathcal{C}}$, the right and left coactions on $M$ are denoted by $\rho^{M}$ and ${ }^{M} \rho$, respectively. If $M, N \in{ }^{\mathcal{C}} \mathbf{M}^{\mathcal{C}}$, then ${ }^{\mathcal{C}} \operatorname{Hom}^{\mathcal{C}}(M, N)$, ${ }_{A} \operatorname{Hom}^{\mathcal{C}}(M, N)$, and ${ }^{\mathcal{C}} \operatorname{Hom}_{A}(M, N)$ denote the set of all $(\mathcal{C}, \mathcal{C})$-bicomodule maps, the set of all right $\mathcal{C}$-comodule left $A$-module maps, and the set of all left $\mathcal{C}$-comodule right $A$-module maps from $M$ to $N$, respectively. If $X, Y \in{ }_{A} \mathbf{M}_{A}$, then the set of all $(A, A)$-bimodule maps from $X$ to $Y$ is denoted by ${ }_{A} \operatorname{Hom}_{A}(X, Y)$. The identity map of a set $X$ is denoted by $I_{X}$.

The notion of a coderivation was introduced in the context of cohomology theory of coalgebras in [3] and [7]. To state the definition of a coderivation, we prepare the next notation.

Definition 1.1. For $M \in{ }^{\mathcal{C}} \mathbf{M}^{\mathcal{C}}$, we define the $R$-linear map

$$
T_{M}:{ }_{A} \operatorname{Hom}_{A}(M, \mathcal{C})^{3} \rightarrow{ }_{A} \operatorname{Hom}_{A}\left(M, \mathcal{C} \otimes_{A} \mathcal{C}\right)
$$

by setting $T_{M}(f, g, h)=\Delta \circ f-\left(g \otimes I_{\mathcal{C}}\right) \circ \rho^{M}-\left(I_{\mathcal{C}} \otimes h\right) \circ{ }^{M} \rho$.

According to [3], [7], and [4], a map $f$ in ${ }_{A} \operatorname{Hom}_{A}(M, \mathcal{C})$ is called a coderivation if $T_{M}(f, f, f)=0$. The set of all coderivations from $M$ to $\mathcal{C}$ is denoted by 
$\operatorname{Coder}(M, \mathcal{C})$. This notion was generalized in [9]. A map $f$ in ${ }_{A} \operatorname{Hom}_{A}(M, \mathcal{C})$ is called a generalized coderivation if $T_{M}(f, f, f)$ is a $(\mathcal{C}, \mathcal{C})$-bicomodule map. The set of all generalized coderivations from $M$ to $\mathcal{C}$ is denoted by $\operatorname{GCoder}(M, \mathcal{C})$. It was proved in $[9$, Lemma $2.2(1)]$ that every generalized coderivation $f$ induces coderivations $d$ and $d^{\prime}$ such that $T_{M}(f, f, d)=0$ and $T_{M}\left(f, d^{\prime}, f\right)=0$. This situation led us to study a triplet $(f, g, h)$ satisfying $T_{M}(f, g, h)=0$.

Definition 1.2. For $M \in{ }^{\mathcal{C}} \mathbf{M}^{\mathcal{C}}$, a triplet $(f, g, h)$ in ${ }_{A} \operatorname{Hom}_{A}(M, \mathcal{C})^{3}$ is called a triple coderivation if $T_{M}(f, g, h)=0$. The set of all triple coderivations is denoted by $\operatorname{TCoder}(M, \mathcal{C})$.

The purpose of this paper is to determine the structure of $\operatorname{TCoder}(M, \mathcal{C})$. The next is the main result of this paper.

Theorem 1.3. Let $\mathcal{C}$ be an $A$-coring and $M \in{ }^{\mathcal{C}} \mathbf{M}^{\mathcal{C}}$. Then the $R$-linear map

$$
\Phi_{M}: \operatorname{TCoder}(M, \mathcal{C}) \rightarrow \operatorname{Coder}(M, \mathcal{C}) \times{ }_{A} \operatorname{Hom}^{\mathcal{C}}(M, \mathcal{C}) \times{ }^{\mathcal{C}} \operatorname{Hom}_{A}(M, \mathcal{C})
$$

defined by $\Phi_{M}(f, g, h)=(g+h-f, f-h, f-g)$ is an isomorphism, and the inverse map is given by $\left(\Phi_{M}\right)^{-1}(d, \varphi, \psi)=(d+\varphi+\psi, d+\varphi, d+\psi)$.

This theorem yields that each component of a triple coderivation is a generalized coderivation. As an application of Theorem 1.3, we investigate symmetrical properties of triple coderivations such as the condition for both $(f, g, h)$ and $(f, h, g)$ to be triple coderivations. Furthermore we investigate the Lie algebra structure of $\operatorname{TCoder}(\mathcal{C}, \mathcal{C})$. In final section we represent triple coderivations by triple derivations of algebras.

\section{Proof of Theorem 1.3}

We prepare some notations and some results to prove Theorem 1.3. Let $\mathcal{C}$ be an $A$-coring and $M \in{ }^{\mathcal{C}} \mathbf{M}^{\mathcal{C}}$. Let $\varepsilon^{M}$ denote the composition map

$$
M \otimes_{A} \mathcal{C} \stackrel{I_{M} \otimes \varepsilon}{\longrightarrow} M \otimes_{A} A \stackrel{\text { canonical isom. }}{\longrightarrow} M
$$

and ${ }^{M} \varepsilon$ denote the composition map

$$
\mathcal{C} \otimes_{A} M \stackrel{\varepsilon \otimes I_{M}}{\longrightarrow} A \otimes_{A} M \stackrel{\text { canonical isom. }}{\longrightarrow} M \text {. }
$$


Usually $\varepsilon^{M}$ and ${ }^{M_{\varepsilon}} \varepsilon$ are represented by $I_{M} \otimes \varepsilon$ and $\varepsilon \otimes I_{M}$, respectively. The following three $R$-isomorphisms are well-known.

$$
\begin{aligned}
& \mathfrak{R}_{M}:{ }_{A} \operatorname{Hom}_{A}(M, A) \rightarrow{ }_{A} \operatorname{Hom}^{\mathcal{C}}(M, \mathcal{C}) \\
& \mathfrak{L}_{M}:{ }_{A} \operatorname{Hom}_{A}(M, A) \rightarrow{ }^{\mathcal{C}} \operatorname{Hom}_{A}(M, \mathcal{C}) \\
& \mathfrak{T}_{M}:{ }_{A} \operatorname{Hom}_{A}(M, A) \rightarrow{ }^{\mathcal{C}} \operatorname{Hom}^{\mathcal{C}}\left(M, \mathcal{C} \otimes{ }_{A} \mathcal{C}\right)
\end{aligned}
$$

For $\xi \in{ }_{A} \operatorname{Hom}_{A}(M, A), \mathfrak{R}_{M}(\xi)$ is the composition map

$$
M \stackrel{\rho^{M}}{\longrightarrow} M \otimes_{A} \mathcal{C} \stackrel{\xi \otimes I_{\mathcal{C}}}{\longrightarrow} A \otimes_{A} \mathcal{C} \stackrel{\text { canonical isom. }}{\longrightarrow} \mathcal{C}
$$

$\mathfrak{L}_{M}(\xi)$ is the composition map

$$
M \stackrel{{ }^{M}}{\longrightarrow} \mathcal{C} \otimes_{A} M \stackrel{I_{\mathcal{C}} \otimes \xi}{\longrightarrow} \mathcal{C} \otimes_{A} A \stackrel{\text { canonical isom. }}{\longrightarrow} \mathcal{C}
$$

and $\mathfrak{T}_{M}(\xi)$ is the composition map

$$
M \stackrel{\left({ }^{M} \rho \otimes I_{\mathcal{C}}\right) \circ \rho^{M}}{\longrightarrow} \mathcal{C} \otimes_{A} M \otimes_{A} \mathcal{C} \stackrel{I_{\mathcal{C}} \otimes \xi \otimes I_{\mathcal{C}}}{\longrightarrow} \mathcal{C} \otimes_{A} A \otimes_{A} \mathcal{C} \stackrel{\text { canonical isom. }}{\longrightarrow} \mathcal{C} \otimes_{A} \mathcal{C} .
$$

The inverse maps are given by $\left(\mathfrak{R}_{M}\right)^{-1}(f)=\varepsilon \circ f,\left(\mathfrak{L}_{M}\right)^{-1}(g)=\varepsilon \circ g$, and $\left(\mathfrak{T}_{M}\right)^{-1}(h)=\varepsilon^{(2)} \circ h$, where $\varepsilon^{(2)}$ is the composition map

$$
\mathcal{C} \otimes{ }_{A} \mathcal{C} \stackrel{\varepsilon \otimes \varepsilon}{\longrightarrow} A \otimes_{A} A \stackrel{\text { canonical isom. }}{\longrightarrow} A .
$$

Usually, $\mathfrak{R}_{M}(\xi), \mathfrak{L}_{M}(\xi)$, and $\mathfrak{T}_{M}(\xi)$ are represented by $\left(\xi \otimes I_{\mathcal{C}}\right) \circ \rho^{M},\left(I_{\mathcal{C}} \otimes \xi\right) \circ M_{\rho}$, and $\left(I_{\mathcal{C}} \otimes \xi \otimes I_{\mathcal{C}}\right) \circ\left({ }^{M} \rho \otimes I_{\mathcal{C}}\right) \circ \rho^{M}$, respectively.

Lemma 2.1. For any $M \in{ }^{\mathcal{C}} \mathbf{M}^{\mathcal{C}}$ and $f, g, h \in{ }_{A} \operatorname{Hom}_{A}(M, \mathcal{C})$, the following hold.

(1) $\varepsilon^{\mathcal{C}} \circ T_{M}(f, g, h)=f-g-\mathfrak{L}_{M}(\varepsilon \circ h)$.

(2) $\mathcal{C}_{\varepsilon} \circ T_{M}(f, g, h)=f-\mathfrak{R}_{M}(\varepsilon \circ g)-h$.

(3) $T_{M}(f, f, 0)=0$ if and only if $f \in{ }_{A} \operatorname{Hom}^{\mathcal{C}}(M, \mathcal{C})$.

(4) $T_{M}(f, 0, f)=0$ if and only if $f \in{ }^{\mathcal{C}} \operatorname{Hom}_{A}(M, \mathcal{C})$.

(5) $T_{M}(0, f,-f)=0$ if and only if $f \in{ }^{\mathcal{C}} \operatorname{Hom}^{\mathcal{C}}(M, \mathcal{C})$.

Proof. (1) We can see that $\varepsilon^{\mathcal{C}} \circ \Delta \circ f=f, \varepsilon^{\mathcal{C}} \circ\left(g \otimes I_{\mathcal{C}}\right) \circ \rho^{M}=g \circ \varepsilon^{M} \circ \rho^{M}=g$, and $\varepsilon^{\mathcal{C}} \circ\left(I_{\mathcal{C}} \otimes h\right) \circ{ }^{M} \rho=\mathfrak{L}_{M}(\varepsilon \circ h)$. Hence $\varepsilon^{\mathcal{C}} \circ T_{M}(f, g, h)=f-g-\mathfrak{L}_{M}(\varepsilon \circ h)$.

(2) is similar to (1).

(3) and (4) are clear by definition.

(5) If $f \in{ }^{\mathcal{C}} \operatorname{Hom}^{\mathcal{C}}(M, \mathcal{C})$, then we have

$$
T_{M}(0, f,-f)=T_{M}(f, f, 0)-T_{M}(f, 0, f)=0
$$

by (3) and (4). Conversely suppose that $T_{M}(0, f,-f)=0$. Then by (1) we have

$$
0=\varepsilon^{\mathcal{C}} \circ T_{M}(0, f,-f)=-f+\mathfrak{L}_{M}(\varepsilon \circ f),
$$


and hence $f \in{ }^{\mathcal{C}} \operatorname{Hom}_{A}(M, \mathcal{C})$. Similarly we have $f \in{ }_{A} \operatorname{Hom}^{\mathcal{C}}(M, \mathcal{C})$ by $(2)$.

The following two lemmas were proved in [9] for coalgebras. For the sake of completeness we give proofs.

Lemma 2.2. For any $M \in{ }^{\mathcal{C}} \mathbf{M}^{\mathcal{C}}$ and $f \in{ }_{A} \operatorname{Hom}_{A}(M, \mathcal{C})$, the following conditions are equivalent.

(1) $f \in \operatorname{GCoder}(M, \mathcal{C})$

(2) $T_{M}(f, f, f)+\mathfrak{T}_{M}(\varepsilon \circ f)=0$

(3) $f-\mathfrak{R}_{M}(\varepsilon \circ f) \in \operatorname{Coder}(M, \mathcal{C})$

(4) $f-\mathfrak{L}_{M}(\varepsilon \circ f) \in \operatorname{Coder}(M, \mathcal{C})$

Proof. $(1) \Rightarrow(2)$ It is easy to see that

$\varepsilon^{(2)} \circ \Delta=\varepsilon \quad$ and $\quad \varepsilon^{(2)} \circ\left(f \otimes I_{\mathcal{C}}\right) \circ \rho^{M}=\varepsilon^{(2)} \circ\left(I_{\mathcal{C}} \otimes f\right) \circ{ }^{M} \rho=\varepsilon \circ f$.

It follows that $\left(\mathfrak{T}_{M}\right)^{-1}\left(T_{M}(f, f, f)\right)=-\varepsilon \circ f$. Hence, $T_{M}(f, f, f)=-\mathfrak{T}_{M}(\varepsilon \circ f)$.

$(2) \Rightarrow(1)$ is clear.

$(2) \Leftrightarrow(3)$ We set $\varphi=\mathfrak{R}_{M}(\varepsilon \circ f)$ and $d=f-\varphi$. Since $\varphi \in{ }_{A} \operatorname{Hom}^{\mathcal{C}}(M, \mathcal{C})$, we have $T_{M}(\varphi, \varphi, 0)=0$. By the definitions of $\mathfrak{R}_{M}$ and $\mathfrak{T}_{M}$, we have

$$
\mathfrak{T}_{M}(\varepsilon \circ f)=\left(I_{\mathcal{C}} \otimes \varphi\right) \circ{ }^{M} \rho=-T_{M}(0,0, \varphi) .
$$

Therefore we see that

$$
T_{M}(f, f, f)=T_{M}(d, d, d)+T_{M}(\varphi, \varphi, 0)+T_{M}(0,0, \varphi)=T_{M}(d, d, d)-\mathfrak{T}_{M}(\varepsilon \circ f) .
$$

Hence $T_{M}(f, f, f)+\mathfrak{T}_{M}(\varepsilon \circ f)=0$ is equivalent to $d \in \operatorname{Coder}(M, \mathcal{C})$.

$(2) \Leftrightarrow(4)$ is similar to $(2) \Leftrightarrow(3)$.

Lemma 2.3. For any $M \in{ }^{\mathcal{C}} \mathbf{M}^{\mathcal{C}}$, the following hold.

(1) $\operatorname{Coder}(M, \mathcal{C})=\{f \in \operatorname{GCoder}(M, \mathcal{C}) \mid \varepsilon \circ f=0\}$

(2) $\operatorname{GCoder}(M, \mathcal{C})=\operatorname{Coder}(M, \mathcal{C}) \oplus{ }_{A} \operatorname{Hom}^{\mathcal{C}}(M, \mathcal{C})$

(3) $\operatorname{GCoder}(M, \mathcal{C})=\operatorname{Coder}(M, \mathcal{C}) \oplus{ }^{\mathcal{C}} \operatorname{Hom}_{A}(M, \mathcal{C})$

Proof. (1) Since $\operatorname{Coder}(M, \mathcal{C}) \subseteq \operatorname{GCoder}(M, \mathcal{C})$, the assertion is clear by the condition (2) of Lemma 2.2.

(2) If $\varphi \in{ }_{A} \operatorname{Hom}^{\mathcal{C}}(M, \mathcal{C})$, then

$$
T_{M}(\varphi, \varphi, \varphi)=T_{M}(\varphi, \varphi, 0)+T_{M}(0,0, \varphi)=-\left(I_{\mathcal{C}} \otimes \varphi\right) \circ{ }^{M} \rho
$$

is a $(\mathcal{C}, \mathcal{C})$-bicomodule map, and hence $\varphi \in \operatorname{GCoder}(M, \mathcal{C})$. Therefore we have $\operatorname{Coder}(M, \mathcal{C})+{ }_{A} \operatorname{Hom}^{\mathcal{C}}(M, \mathcal{C}) \subseteq \operatorname{GCoder}(M, \mathcal{C})$. By (3) of Lemma 2.2 we have

$$
\operatorname{GCoder}(M, \mathcal{C})=\operatorname{Coder}(M, \mathcal{C})+{ }_{A} \operatorname{Hom}^{\mathcal{C}}(M, \mathcal{C}) .
$$


Let $d \in \operatorname{Coder}(M, \mathcal{C}) \cap{ }_{A} \operatorname{Hom}^{\mathcal{C}}(M, \mathcal{C})$. Then, using Lemma 2.1, we see that

$$
0={ }^{\mathcal{C}} \varepsilon \circ\left(T_{M}(d, d, d)-T_{M}(d, d, 0)\right)={ }^{\mathcal{C}} \varepsilon \circ T_{M}(0,0, d)=-d .
$$

Hence, $\operatorname{Coder}(M, \mathcal{C}) \cap{ }_{A} \operatorname{Hom}^{\mathcal{C}}(M, \mathcal{C})=0$.

(3) is similar to (2).

Proof of Theorem 1.3. Let $(f, g, h) \in \operatorname{TCoder}(M, \mathcal{C})$. By $(1)$ and (2) of Lemma 2.1 we have

$$
\begin{aligned}
& f=g+\mathfrak{L}_{M}(\varepsilon \circ h), \quad \text { and } \\
& f=h+\mathfrak{R}_{M}(\varepsilon \circ g) .
\end{aligned}
$$

Therefore we see that

$$
\begin{aligned}
T_{M}(f, f, f) & =T_{M}(f, g, h)+T_{M}\left(0, \mathfrak{L}_{M}(\varepsilon \circ h), \mathfrak{R}_{M}(\varepsilon \circ g)\right) \\
& =-\left(\mathfrak{L}_{M}(\varepsilon \circ h) \otimes I_{\mathcal{C}}\right) \circ \rho^{M}-\left(I_{\mathcal{C}} \otimes \mathfrak{R}_{M}(\varepsilon \circ g)\right) \circ{ }^{M_{\rho}} .
\end{aligned}
$$

It follows that $T_{M}(f, f, f)$ is a $(\mathcal{C}, \mathcal{C})$-bicomodule map, and hence $f \in \operatorname{GCoder}(M, \mathcal{C})$. By the equation (2.1), $g$ also belongs to $\operatorname{GCoder}(M, \mathcal{C})$. By the equation $(2.2)$, we have $g+h-f=g-\mathfrak{R}_{M}(\varepsilon \circ g)$, which belongs to $\operatorname{Coder}(M, \mathcal{C})$ by Lemma 2.2. Together this result with equations (2.1) and (2.2), we can define the $R$-linear map

$$
\Phi_{M}: \operatorname{TCoder}(M, \mathcal{C}) \rightarrow \operatorname{Coder}(M, \mathcal{C}) \times{ }_{A} \operatorname{Hom}^{\mathcal{C}}(M, \mathcal{C}) \times{ }^{\mathcal{C}} \operatorname{Hom}_{A}(M, \mathcal{C})
$$

by setting $\Phi_{M}(f, g, h)=(g+h-f, f-h, f-g)$.

Conversely, for any $d \in \operatorname{Coder}(M, \mathcal{C}), \varphi \in{ }_{A} \operatorname{Hom}^{\mathcal{C}}(M, \mathcal{C})$, and $\psi \in{ }^{\mathcal{C}} \operatorname{Hom}_{A}(M, \mathcal{C})$, we see that

$$
T_{M}(d+\varphi+\psi, d+\varphi, d+\psi)=T_{M}(d, d, d)+T_{M}(\varphi, \varphi, 0)+T_{M}(\psi, 0, \psi)=0,
$$

and hence $(d+\varphi+\psi, d+\varphi, d+\psi) \in \operatorname{TCoder}(M, \mathcal{C})$. Thus we can define the map

$$
\Psi_{M}: \operatorname{Coder}(M, \mathcal{C}) \times{ }_{A} \operatorname{Hom}^{\mathcal{C}}(M, \mathcal{C}) \times{ }^{\mathcal{C}} \operatorname{Hom}_{A}(M, \mathcal{C}) \rightarrow \operatorname{TCoder}(M, \mathcal{C})
$$

by setting $\Psi_{M}(d, \varphi, \psi)=(d+\varphi+\psi, d+\varphi, d+\psi)$. It is easy to see that $\Psi_{M}$ is the inverse map of $\Phi_{M}$. Hence $\Phi_{M}$ is an isomorphism.

The proof of Theorem 1.3 shows the next.

Corollary 2.4. The map $\Phi_{M}$ given in Theorem 1.3 satisfies

$$
\Phi_{M}(f, g, h)=\left(f-\mathfrak{R}_{M}(\varepsilon \circ g)-\mathfrak{L}_{M}(\varepsilon \circ h), \mathfrak{R}_{M}(\varepsilon \circ g), \mathfrak{L}_{M}(\varepsilon \circ h)\right)
$$

and $f-\mathfrak{R}_{M}(\varepsilon \circ g)-\mathfrak{L}_{M}(\varepsilon \circ h)=g-\mathfrak{R}_{M}(\varepsilon \circ g)=h-\mathfrak{L}_{M}(\varepsilon \circ h)$. 
Corollary 2.5. The R-module $\operatorname{TCoder}(M, \mathcal{C})$ is a subdirect product of three copies of $\operatorname{GCoder}(M, \mathcal{C})$, i.e., all components of a triple coderivation are generalized coderivations and every generalized coderivation appears in each component of triple coderivations.

Proof. By Theorem 1.3 all components of triple coderivations belong to

$$
\operatorname{Coder}(M, \mathcal{C})+{ }_{A} \operatorname{Hom}^{\mathcal{C}}(M, \mathcal{C})+{ }^{\mathcal{C}} \operatorname{Hom}_{A}(M, \mathcal{C}) .
$$

Hence they are generalized coderivations by Lemma 2.3. Conversely let $f \in$ $\operatorname{GCoder}(M, \mathcal{C})$. Then, by Lemma $2.3, f$ can be written as $f=d+\varphi=d^{\prime}+\psi$ with some $d, d^{\prime} \in \operatorname{Coder}(M, \mathcal{C}), \varphi \in{ }_{A} \operatorname{Hom}^{\mathcal{C}}(M, \mathcal{C})$, and $\psi \in{ }^{\mathcal{C}} \operatorname{Hom}_{A}(M, \mathcal{C})$. Theorem 1.3 shows that $(f, f, d)=\left(\Phi_{M}\right)^{-1}(d, \varphi, 0)$ and $\left(f, d^{\prime}, f\right)=\left(\Phi_{M}\right)^{-1}\left(d^{\prime}, 0, \psi\right)$ are triple coderivations.

Finally we characterize a triplet $(f, g, h)$ such that $T_{M}(f, g, h)$ is a $(\mathcal{C}, \mathcal{C})$-bicomodule map.

Proposition 2.6. Let $\mathcal{C}$ be an A-coring, $M \in{ }^{\mathcal{C}} \mathbf{M}^{\mathcal{C}}$, and $f, g, h \in{ }_{A} \operatorname{Hom}_{A}(M, \mathcal{C})$. Then $T_{M}(f, g, h)$ is a $(\mathcal{C}, \mathcal{C})$-bicomodule map if and only if $f \in \operatorname{GCoder}(M, \mathcal{C})$, $f-g \in{ }^{\mathcal{C}} \operatorname{Hom}_{A}(M, \mathcal{C})$, and $f-h \in{ }_{A} \operatorname{Hom}^{\mathcal{C}}(M, \mathcal{C})$ hold. When this is the case, $g$ and $h$ also belong to $\operatorname{GCoder}(M, \mathcal{C})$.

Proof. Suppose that $T_{M}(f, g, h)$ is a $(\mathcal{C}, \mathcal{C})$-bicomodule map. Then by Lemma 2.1 (1) we have $\varepsilon^{\mathcal{C}} \circ T_{M}(f, g, h)=f-g-\mathfrak{L}_{M}(\varepsilon \circ h)$. Since $\varepsilon^{\mathcal{C}}$ is a left $\mathcal{C}$-comodule map, so is $f-g$. Similarly $f-h$ is a right $\mathcal{C}$-comodule map. Therefore

$$
T_{M}(0, f-g, f-h)=-\left((f-g) \otimes I_{C}\right) \circ \rho^{M}-\left(I_{\mathcal{C}} \otimes(f-h)\right) \circ{ }^{M} \rho
$$

is a $(\mathcal{C}, \mathcal{C})$-bicomodule map. Hence $T_{M}(f, f, f)=T_{M}(f, g, h)+T_{M}(0, f-g, f-h)$ is a $(\mathcal{C}, \mathcal{C})$-bicomodule map, which means that $f \in \operatorname{GCoder}(M, \mathcal{C})$. Since $f-$ $g \in{ }^{\mathcal{C}} \operatorname{Hom}_{A}(M, \mathcal{C}), g$ belongs to $\operatorname{GCoder}(M, \mathcal{C})$ by Lemma 2.3 (3). Similarly $h$ belongs to $\operatorname{GCoder}(M, \mathcal{C})$. Conversely if $f \in \operatorname{GCoder}(M, \mathcal{C}), f-g \in{ }^{\mathcal{C}} \operatorname{Hom}_{A}(M, \mathcal{C})$, and $f-h \in{ }_{A} \operatorname{Hom}^{\mathcal{C}}(M, \mathcal{C})$, then $T_{M}(f, f, f)$ and $T_{M}(0, f-g, f-h)$ are $(\mathcal{C}, \mathcal{C})$ bicomodule maps, and hence $T_{M}(f, g, h)=T_{M}(f, f, f)-T_{M}(0, f-g, f-h)$ is a $(\mathcal{C}, \mathcal{C})$-bicomodule map.

\section{Symmetrical properties of triple coderivations}

In this section we investigate symmetrical properties of triple coderivations. First we introduce the next. 
Definition 3.1. Let $M \in{ }^{\mathcal{C}} \mathbf{M}^{\mathcal{C}}$. A map $f$ in ${ }_{A} \operatorname{Hom}_{A}(M, \mathcal{C})$ is called a symmetric generalized coderivation if there exists $\varphi \in{ }^{\mathcal{C}} \operatorname{Hom}^{\mathcal{C}}(M, \mathcal{C})$ such that $T_{M}(f, f, f)=$ $\Delta \circ \varphi$. The set of all symmetric generalized coderivations from $M$ to $\mathcal{C}$ is denoted by $\operatorname{SGCoder}(M, \mathcal{C})$.

Obviously every symmetric generalized coderivation is a generalized coderivation.

Lemma 3.2. For any $M \in{ }^{\mathcal{C}} \mathbf{M}^{\mathcal{C}}$ and $f \in \operatorname{GCoder}(M, \mathcal{C})$, the following conditions are equivalent.

(1) $f \in \operatorname{SGCoder}(M, \mathcal{C})$

(2) There exists $\varphi \in{ }^{\mathcal{C}} \operatorname{Hom}^{\mathcal{C}}(M, \mathcal{C})$ such that $\varepsilon \circ f=\varepsilon \circ \varphi$.

(3) $\mathfrak{R}_{M}(\varepsilon \circ f)=\mathfrak{L}_{M}(\varepsilon \circ f)$

Proof. $(1) \Rightarrow(2)$ There exists $\varphi \in{ }^{\mathcal{C}} \operatorname{Hom}^{\mathcal{C}}(M, \mathcal{C})$ such that $T_{M}(f, f, f)=\Delta \circ \varphi$. It follows that $\varepsilon^{\mathcal{C}} \circ T_{M}(f, f, f)=\varphi$. On the other hand, by Lemma 2.1 (1), we have

$$
\varepsilon^{\mathcal{C}} \circ T_{M}(f, f, f)=f-f-\mathfrak{L}_{M}(\varepsilon \circ f)=-\mathfrak{L}_{M}(\varepsilon \circ f) .
$$

It follows that $\varphi=-\mathfrak{L}_{M}(\varepsilon \circ f)$. Hence $\varepsilon \circ f=\varepsilon \circ \mathfrak{L}_{M}(\varepsilon \circ f)=\varepsilon \circ(-\varphi)$.

$(2) \Rightarrow(3)$ There exists $\varphi \in{ }^{\mathcal{C}} \operatorname{Hom}^{\mathcal{C}}(M, \mathcal{C})$ such that $\varepsilon \circ f=\varepsilon \circ \varphi$. Hence we have $\mathfrak{R}_{M}(\varepsilon \circ f)=\varphi=\mathfrak{L}_{M}(\varepsilon \circ f)$.

$(3) \Rightarrow(1)$ By definition we have $\mathfrak{T}_{M}(\varepsilon \circ f)=\left(I_{\mathcal{C}} \otimes \mathfrak{R}_{M}(\varepsilon \circ f)\right) \circ{ }^{M} \rho$. Since $\mathfrak{R}_{M}(\varepsilon \circ f)=\mathfrak{L}(\varepsilon \circ f) \in{ }^{\mathcal{C}} \operatorname{Hom}^{\mathcal{C}}(M, \mathcal{C})$, we have $\left(I_{\mathcal{C}} \otimes \mathfrak{R}_{M}(\varepsilon \circ f)\right) \circ{ }^{M} \rho=\Delta \circ \mathfrak{R}_{M}(\varepsilon \circ f)$. By Lemma 2.2 we have $T_{M}(f, f, f)=\Delta \circ\left(-\mathfrak{R}_{M}(\varepsilon \circ f)\right)$.

In virtue of Lemmas 2.2, 2.3, and 3.2, we get the next.

Corollary 3.3. $\operatorname{SGCoder}(M, \mathcal{C})=\operatorname{Coder}(M, \mathcal{C}) \oplus{ }^{\mathcal{C}} \operatorname{Hom}^{\mathcal{C}}(M, \mathcal{C})$ for all $M \in{ }^{\mathcal{C}} \mathbf{M}^{\mathcal{C}}$.

Theorem 3.4. Let $\mathcal{C}$ be an $A$-coring, $M \in{ }^{\mathcal{C}} \mathbf{M}^{\mathcal{C}}$, and $(f, g, h) \in \operatorname{TCoder}(M, \mathcal{C})$. Then the following hold.

(1) $(f, h, g) \in \mathrm{TCoder}(M, \mathcal{C})$ if and only if $g, h \in \operatorname{SGCoder}(M, \mathcal{C})$. When this is the case, $f$ also belongs to $\operatorname{SGCoder}(M, \mathcal{C})$.

$(2)(h, g, f) \in \operatorname{TCoder}(M, \mathcal{C})$ if and only if $g \in \operatorname{SGCoder}(M, \mathcal{C})$ and $2 f=2 h$.

(3) $(g, f, h) \in \operatorname{TCoder}(M, \mathcal{C})$ if and only if $h \in \operatorname{SGCoder}(M, \mathcal{C})$ and $2 f=2 g$.

(4) $(h, f, g) \in \operatorname{TCoder}(M, \mathcal{C})$ if and only if $g, h \in \operatorname{SGCoder}(M, \mathcal{C})$ and $2 f=2 h$. When this is the case, $f$ also belongs to $\operatorname{SGCoder}(M, \mathcal{C})$.

$(5)(g, h, f) \in \operatorname{TCoder}(M, \mathcal{C})$ if and only if $g, h \in \operatorname{SGCoder}(M, \mathcal{C})$ and $2 f=2 g$. When this is the case, $f$ also belongs to $\operatorname{SGCoder}(M, \mathcal{C})$. 
Proof. Set $d=g+h-f, \varphi=f-h$, and $\psi=f-g$. Then by Theorem 1.3 we have $d \in \operatorname{Coder}(M, \mathcal{C}), \varphi \in{ }_{A} \operatorname{Hom}^{\mathcal{C}}(M, \mathcal{C}), \psi \in{ }^{\mathcal{C}} \operatorname{Hom}_{A}(M, \mathcal{C})$, and $(f, g, h)=$ $(d+\varphi+\psi, d+\varphi, d+\psi)$.

(1) Suppose that $(f, h, g) \in \operatorname{TCoder}(M, \mathcal{C})$. Then by Theorem 1.3 we have $\psi=f-g \in{ }_{A} \operatorname{Hom}^{\mathcal{C}}(M, \mathcal{C})$ and $\varphi=f-h \in{ }^{\mathcal{C}} \operatorname{Hom}_{A}(M, \mathcal{C})$. It follows that $\varphi$, $\psi \in{ }^{\mathcal{C}} \operatorname{Hom}^{\mathcal{C}}(M, \mathcal{C})$. Hence $f, g, h \in \operatorname{SGCoder}(M, \mathcal{C})$ by Corollary 3.3. Conversely suppose that $g, h \in \operatorname{SGCoder}(M, \mathcal{C})$. Since $g=d+\varphi$ and $h=d+\psi$, both $\varphi$ and $\psi$ belong to ${ }^{\mathcal{C}} \operatorname{Hom}^{\mathcal{C}}(M, \mathcal{C})$ by Corollary 3.3 and Lemma 2.3. Hence $(f, h, g)=$ $\left(\Phi_{M}\right)^{-1}(d, \psi, \varphi) \in \operatorname{TCoder}(M, \mathcal{C})$.

(2) Suppose that $(h, g, f) \in \operatorname{TCoder}(M, \mathcal{C})$. Then by Theorem 1.3 we have $d^{\prime}=g+f-h \in \operatorname{Coder}(M, \mathcal{C}), \varphi^{\prime}=h-f \in{ }_{A} \operatorname{Hom}^{\mathcal{C}}(M, \mathcal{C})$, and $\psi^{\prime}=h-g \in$ ${ }^{\mathcal{C}} \operatorname{Hom}_{A}(M, \mathcal{C})$. Since $\varphi=f-h=\psi-\psi^{\prime}$, we have $\varphi \in{ }^{\mathcal{C}} \operatorname{Hom}^{\mathcal{C}}(M, \mathcal{C})$. Hence $g \in \operatorname{SGCoder}(M, \mathcal{C})$. Furthermore $d^{\prime}-d=2(f-h)=2 \varphi$ belongs to $\operatorname{Coder}(M, \mathcal{C}) \cap$ ${ }_{A} \operatorname{Hom}^{\mathcal{C}}(M, \mathcal{C})$. By Lemma $2.3(2)$ we have $2 \varphi=0$, and hence $2 f=2 h$. Conversely suppose that $g \in \operatorname{SGCoder}(M, \mathcal{C})$ and $2 f=2 h$. Then we have $\varphi \in{ }^{\mathcal{C}} \operatorname{Hom}^{\mathcal{C}}(M, \mathcal{C})$ and $2 \varphi=0$. Hence $(h, g, f)=\left(\Phi_{M}\right)^{-1}(d, \varphi, \varphi+\psi) \in \operatorname{TCoder}(M, \mathcal{C})$.

(3) is similar to (2).

(4) Suppose that $(h, f, g) \in \operatorname{TCoder}(M, \mathcal{C})$. Then we have $d^{\prime}=f+g-h \in$ $\operatorname{Coder}(M, \mathcal{C}), \varphi^{\prime}=h-g \in{ }_{A} \operatorname{Hom}^{\mathcal{C}}(M, \mathcal{C})$, and $\psi^{\prime}=h-f \in{ }^{\mathcal{C}} \operatorname{Hom}_{A}(M, \mathcal{C})$. Since $\varphi=f-h=-\psi^{\prime}$ and $\psi=f-g=\varphi+\varphi^{\prime}$, we have $\varphi, \psi \in{ }^{\mathcal{C}} \operatorname{Hom}^{\mathcal{C}}(M, \mathcal{C})$. Hence $f, g, h \in \operatorname{SGCoder}(M, C)$. Furthermore $d^{\prime}-d=2(f-h)=2 \varphi$ belongs to $\operatorname{Coder}(M, \mathcal{C}) \cap{ }_{A} \operatorname{Hom}^{\mathcal{C}}(M, \mathcal{C})$. By Lemma $2.3(2)$ we have $2 \varphi=0$, and hence $2 f=2 h$. Conversely suppose that $g, h \in \operatorname{SGCoder}(M, \mathcal{C})$ and $2 f=2 h$. Then we have $\varphi, \psi \in{ }^{\mathcal{C}} \operatorname{Hom}^{\mathcal{C}}(M, \mathcal{C})$ and $2 \varphi=0$. Hence $(h, f, g)=\left(\Phi_{M}\right)^{-1}(d, \varphi+\psi, \varphi) \in$ $\operatorname{TCoder}(M, \mathcal{C})$.

(5) is similar to (4).

Corollary 3.5. For any $M \in{ }^{\mathcal{C}} \mathbf{M}^{\mathcal{C}}$ and $f, g \in{ }_{A} \operatorname{Hom}_{A}(M, \mathcal{C})$, the following hold.

(1) $(f, g, g) \in \operatorname{TCoder}(M, \mathcal{C})$ if and only if $g \in \operatorname{SGCoder}(M, \mathcal{C})$ and $f=g+$ $\mathfrak{R}_{M}(\varepsilon \circ g)$. When this is the case, $f$ also belongs to $\operatorname{SGCoder}(M, \mathcal{C})$.

(2) $(f, g, f) \in \operatorname{TCoder}(M, \mathcal{C})$ if and only if $g \in \operatorname{Coder}(M, \mathcal{C})$ and $f-g \in$ ${ }^{\mathcal{C}} \operatorname{Hom}_{A}(M, \mathcal{C})$.

(3) $(f, f, g) \in \operatorname{TCoder}(M, \mathcal{C})$ if and only if $g \in \operatorname{Coder}(M, \mathcal{C})$ and $f-g \in$ ${ }_{A} \operatorname{Hom}^{\mathcal{C}}(M, \mathcal{C})$.

Proof. (1) If $(f, g, g) \in \operatorname{TCoder}(M, \mathcal{C})$, then we have $f, g \in \operatorname{SGCoder}(M, \mathcal{C})$ by Theorem 3.4 (1) and $f-\mathfrak{R}_{M}(\varepsilon \circ g)-g=0$ by Lemma 2.1 (2). Conversely if $g \in \operatorname{SGCoder}(M, \mathcal{C})$ and $f=g+\mathfrak{R}_{M}(\varepsilon \circ g)$, then we have $\varphi=\mathfrak{R}_{M}(\varepsilon \circ g) \in$ 
${ }^{\mathcal{C}} \operatorname{Hom}^{\mathcal{C}}(M, \mathcal{C})$ by Lemma 3.2 and $d=g-\varphi \in \operatorname{Coder}(M, \mathcal{C})$ by Lemma 2.2, and hence $(f, g, g)=\left(\Phi_{M}\right)^{-1}(d, \varphi, \varphi) \in \operatorname{TCoder}(M, \mathcal{C})$.

(2) If $(f, g, f) \in \operatorname{TCoder}(M, \mathcal{C})$, then we have $f \in \operatorname{GCoder}(M, \mathcal{C})$ by Corollary 2.5 and $f-g-\mathfrak{L}_{M}(\varepsilon \circ f)=0$ by Lemma $2.1(1)$, and hence $g \in \operatorname{Coder}(M, \mathcal{C})$ by Lemma 2.2. Conversely if $g \in \operatorname{Coder}(M, \mathcal{C})$ and $f-g \in{ }^{\mathcal{C}} \operatorname{Hom}_{A}(M, \mathcal{C})$, then we have $(f, g, f)=\left(\Phi_{M}\right)^{-1}(g, 0, f-g) \in \operatorname{TCoder}(M, \mathcal{C})$.

(3) is similar to $(2)$.

\section{Triple inner coderivations}

Let $M \in{ }^{\mathcal{C}} \mathbf{M}^{\mathcal{C}}$. According to [3], [7] and [4], a map in ${ }_{A} \operatorname{Hom}_{A}(M, \mathcal{C})$ of the form $\mathfrak{R}_{M}(\xi)-\mathfrak{L}_{M}(\xi)$ with some $\xi \in{ }_{A} \operatorname{Hom}_{A}(M, \mathcal{C})$ is called an inner coderivation. The set of all inner coderivations from $M$ to $\mathcal{C}$ is denoted by $\operatorname{In} \operatorname{Coder}(M, \mathcal{C})$. According to [9], an element of ${ }_{A} \operatorname{Hom}^{\mathcal{C}}(M, \mathcal{C})+{ }^{\mathcal{C}} \operatorname{Hom}_{A}(M, \mathcal{C})$ is called a generalized inner coderivation. We set $\operatorname{GInCoder}(M, \mathcal{C})={ }_{A} \operatorname{Hom}^{\mathcal{C}}(M, \mathcal{C})+{ }^{\mathcal{C}} \operatorname{Hom}_{A}(M, \mathcal{C})$.

Lemma 4.1. For any $M \in{ }^{\mathcal{C}} \mathbf{M}^{\mathcal{C}}$, the following hold.

(1) $\operatorname{GInCoder}(M, \mathcal{C}) \cap \operatorname{Coder}(M, \mathcal{C})=\operatorname{In} \operatorname{Coder}(M, \mathcal{C})$

(2) $\operatorname{GCoder}(M, \mathcal{C}) / \operatorname{GInCoder}(M, \mathcal{C}) \simeq \operatorname{Coder}(M, \mathcal{C}) / \operatorname{In} \operatorname{Coder}(M, \mathcal{C})$

Proof. (1) It is clear that $\operatorname{InCoder}(M, \mathcal{C}) \subseteq \operatorname{GInCoder}(M, \mathcal{C}) \cap \operatorname{Coder}(M, \mathcal{C})$. Conversely let $d \in \operatorname{GInCoder}(M, \mathcal{C}) \cap \operatorname{Coder}(M, \mathcal{C})$. Then there exist $\varphi \in{ }_{A} \operatorname{Hom}^{\mathcal{C}}(M, \mathcal{C})$ and $\psi \in{ }^{\mathcal{C}} \operatorname{Hom}_{A}(M, \mathcal{C})$ such that $d=\varphi+\psi$. By Lemma 2.3 (1), we have $\varepsilon \circ \varphi+\varepsilon \circ \psi=\varepsilon \circ d=0$. Since $\left(\mathfrak{R}_{M}\right)^{-1}(\varphi)=\varepsilon \circ \varphi$ and $\left(\mathfrak{L}_{M}\right)^{-1}(\psi)=\varepsilon \circ \psi=-\varepsilon \circ \varphi$, we have $d=\mathfrak{R}_{M}(\varepsilon \circ \varphi)-\mathfrak{L}_{M}(\varepsilon \circ \varphi)$. Hence $d \in \operatorname{InCoder}(M, \mathcal{C})$.

(2) Lemma $2.3(2)$ implies that $\operatorname{GCoder}(M, \mathcal{C})=\operatorname{GInCoder}(M, \mathcal{C})+\operatorname{Coder}(M, \mathcal{C})$. Using this and (1), we get the assertion.

Definition 4.2. For $M \in{ }^{\mathcal{C}} \mathbf{M}^{\mathcal{C}}$, we set

$$
\text { TInCoder }(M, \mathcal{C})=\operatorname{TCoder}(M, \mathcal{C}) \cap \operatorname{GInCoder}(M, \mathcal{C})^{3} .
$$

An element in $\operatorname{TInCoder}(M, \mathcal{C})$ is called a triple inner coderivation.

Theorem 4.3. Let $\mathcal{C}$ be an $A$-coring and $M \in{ }^{\mathcal{C}} \mathbf{M}^{\mathcal{C}}$. Then the map $\Phi_{M}$ given in Theorem 1.3 induces the $R$-isomorphism

$$
\operatorname{TInCoder}(M, \mathcal{C}) \simeq \operatorname{InCoder}(M, \mathcal{C}) \times{ }_{A} \operatorname{Hom}^{\mathcal{C}}(M, \mathcal{C}) \times{ }^{\mathcal{C}} \operatorname{Hom}_{A}(M, \mathcal{C}) .
$$

Proof. Let $(f, g, h) \in \operatorname{TCoder}(M, \mathcal{C})$ and set $(d, \varphi, \psi)=\Phi_{M}(f, g, h)$. Then by Theorem 1.3 we have $(f, g, h)=(d+\varphi+\psi, d+\varphi, d+\psi)$. Since $\varphi, \psi \in \operatorname{GInCoder}(M, \mathcal{C})$, $(f, g, h) \in \mathrm{TInCoder}(M, \mathcal{C})$ is equivalent to $d \in \operatorname{GIn} \operatorname{Coder}(M, \mathcal{C})$, which is equivalent to $d \in \operatorname{InCoder}(M, \mathcal{C})$ by Lemma $4.1(1)$. Hence we get the assertion. 
By Theorems 1.3 and 4.3, we get the next.

Corollary 4.4. For any $M \in{ }^{\mathcal{C}} \mathbf{M}^{\mathcal{C}}$,

$$
\operatorname{TCoder}(M, \mathcal{C}) / \operatorname{TInCoder}(M, \mathcal{C}) \simeq \operatorname{Coder}(M, \mathcal{C}) / \operatorname{InCoder}(M, \mathcal{C})
$$

as R-modules.

Remark 4.5. Let $M \in{ }^{\mathcal{C}} \mathbf{M}^{\mathcal{C}}$. The kernel of the R-linear map

$$
{ }_{A} \operatorname{Hom}_{A}(M, A) \ni \xi \mapsto \mathfrak{R}_{M}(\xi)-\mathfrak{L}_{M}(\xi) \in \operatorname{In} \operatorname{Coder}(M, \mathcal{C})
$$

coincides with $\varepsilon \circ^{\mathcal{C}} \operatorname{Hom}^{\mathcal{C}}(M, \mathcal{C})$. It follows that

$$
\operatorname{InCoder}(M, \mathcal{C}) \simeq{ }_{A} \operatorname{Hom}_{A}(M, A) / \varepsilon \circ^{\mathcal{C}} \operatorname{Hom}^{\mathcal{C}}(M, \mathcal{C})
$$

as $R$-modules. Hence the following conditions are equivalent.

(1) $\operatorname{InCoder}(M, \mathcal{C})=0$

(2) ${ }_{A} \operatorname{Hom}^{\mathcal{C}}(M, \mathcal{C})={ }^{\mathcal{C}} \operatorname{Hom}^{\mathcal{C}}(M, \mathcal{C})$

(3) ${ }^{\mathcal{C}} \operatorname{Hom}_{A}(M, \mathcal{C})={ }^{\mathcal{C}} \operatorname{Hom}^{\mathcal{C}}(M, \mathcal{C})$

(4) $\operatorname{GInCoder}(M, \mathcal{C})={ }^{\mathcal{C}} \operatorname{Hom}^{\mathcal{C}}(M, \mathcal{C})$

(5) $\operatorname{GCoder}(M, \mathcal{C})=\operatorname{SGCoder}(M, \mathcal{C})$

\section{Lie algebra structure of $\operatorname{TCoder}(\mathcal{C}, \mathcal{C})$}

In [9] it was proved that $\operatorname{Coder}(\mathcal{C}, \mathcal{C})$ and $\operatorname{GCoder}(\mathcal{C}, \mathcal{C})$ are Lie subalgebras of the Lie algebra $\mathfrak{g l}(\mathcal{C})$ consisting of all $R$-endomorphisms of $\mathcal{C}$. In this section, we investigate a Lie algebra structure of $\operatorname{TCoder}(\mathcal{C}, \mathcal{C})$.

We abbreviate $\operatorname{Coder}(\mathcal{C}, \mathcal{C})$ as $\operatorname{Coder}(\mathcal{C})$. Similarly we use the abbreviations InCoder $(\mathcal{C}), \operatorname{GCoder}(\mathcal{C}), \operatorname{GInCoder}(\mathcal{C})$, $\operatorname{SGCoder}(\mathcal{C})$, TCoder $(\mathcal{C})$, and TInCoder $(\mathcal{C})$. We set ${ }_{A} \operatorname{End}^{\mathcal{C}}(\mathcal{C})={ }_{A} \operatorname{Hom}^{\mathcal{C}}(\mathcal{C}, \mathcal{C}),{ }^{\mathcal{C}} \operatorname{End}_{A}(\mathcal{C})={ }^{\mathcal{C}} \operatorname{Hom}_{A}(\mathcal{C}, \mathcal{C})$, and ${ }^{\mathcal{C}} \operatorname{End}^{\mathcal{C}}(\mathcal{C})=$ ${ }^{\mathcal{C}} \operatorname{Hom}^{\mathcal{C}}(\mathcal{C}, \mathcal{C})$.

Lemma 5.1. TCoder $(\mathcal{C})$ is a Lie subalgebra of the product Lie algebra $\mathfrak{g l}(\mathcal{C})^{3}$.

Proof. Let $(f, g, h),\left(f^{\prime}, g^{\prime}, h^{\prime}\right) \in \operatorname{TCoder}(\mathcal{C})$. We see that

$$
\begin{aligned}
\Delta \circ f \circ f^{\prime} & =\left(g \otimes I_{\mathcal{C}}+I_{\mathcal{C}} \otimes h\right) \circ \Delta \circ f^{\prime} \\
& =\left(g \otimes I_{\mathcal{C}}+I_{\mathcal{C}} \otimes h\right) \circ\left(g^{\prime} \otimes I_{\mathcal{C}}+I_{\mathcal{C}} \otimes h^{\prime}\right) \circ \Delta \\
& =\left(\left(g \circ g^{\prime}\right) \otimes I_{\mathcal{C}}+g \otimes h^{\prime}+g^{\prime} \otimes h+I_{\mathcal{C}} \otimes\left(h \circ h^{\prime}\right)\right) \circ \Delta .
\end{aligned}
$$

Similarly we have

$$
\Delta \circ f^{\prime} \circ f=\left(\left(g^{\prime} \circ g\right) \otimes I_{\mathcal{C}}+g^{\prime} \otimes h+g \otimes h^{\prime}+I_{\mathcal{C}} \otimes\left(h^{\prime} \circ h\right)\right) \circ \Delta .
$$


It follows that

$$
\Delta \circ\left[f, f^{\prime}\right]=\left(\left[g, g^{\prime}\right] \otimes I_{\mathcal{C}}+I_{\mathcal{C}} \otimes\left[h, h^{\prime}\right]\right) \circ \Delta .
$$

Hence $\left(\left[f, f^{\prime}\right],\left[g, g^{\prime}\right],\left[h, h^{\prime}\right]\right) \in \operatorname{TCoder}(\mathcal{C})$.

The next corollary can be proved directly from definition. Here we give a proof using Theorem 1.3 and Lemma 5.1.

Corollary 5.2. In the Lie algebra $\mathfrak{g l}(\mathcal{C})$, the following hold.

(1) $\operatorname{Coder}(\mathcal{C})$ is a Lie subalgebra of $\mathfrak{g l}(\mathcal{C})$.

(2) $\left[\operatorname{Coder}(\mathcal{C}),{ }_{A} \operatorname{End}^{\mathcal{C}}(\mathcal{C})\right] \subseteq{ }_{A} \operatorname{End}^{\mathcal{C}}(\mathcal{C})$

(3) $\left[\operatorname{Coder}(\mathcal{C}),{ }^{\mathcal{C}} \operatorname{End}_{A}(\mathcal{C})\right] \subseteq{ }^{\mathcal{C}} \operatorname{End}_{A}(\mathcal{C})$

(4) $\left[{ }_{A} \operatorname{End}^{\mathcal{C}}(\mathcal{C}),{ }^{\mathcal{C}} \operatorname{End}_{A}(\mathcal{C})\right]=0$

Proof. (1) Let $d, d^{\prime} \in \operatorname{Coder}(\mathcal{C})$. Using the map $\Phi_{\mathcal{C}}$ given in Theorem 1.3, we can easily see that

$$
\Phi_{\mathcal{C}}\left(\left[\left(\Phi_{\mathcal{C}}\right)^{-1}(d, 0,0),\left(\Phi_{\mathcal{C}}\right)^{-1}\left(d^{\prime}, 0,0\right)\right]\right)=\left(\left[d, d^{\prime}\right], 0,0\right) .
$$

Hence $\left[d, d^{\prime}\right] \in \operatorname{Coder}(\mathcal{C})$.

(2) For any $d \in \operatorname{Coder}(\mathcal{C})$ and $\varphi \in{ }_{A} \operatorname{End}^{\mathcal{C}}(\mathcal{C})$, we can see that

$$
\Phi_{\mathcal{C}}\left(\left[\left(\Phi_{\mathcal{C}}\right)^{-1}(d, 0,0),\left(\Phi_{\mathcal{C}}\right)^{-1}(0, \varphi, 0)\right]\right)=(0,[d, \varphi], 0),
$$

and hence $[d, \varphi] \in{ }_{A} \operatorname{End}^{\mathcal{C}}(\mathcal{C})$.

(3) is similar to (2).

(4) Let $\varphi \in{ }_{A} \operatorname{End}^{\mathcal{C}}(\mathcal{C})$ and $\psi \in{ }^{\mathcal{C}} \operatorname{End}_{A}(\mathcal{C})$. We can see that

$$
\Phi_{\mathcal{C}}\left(\left[\left(\Phi_{\mathcal{C}}\right)^{-1}(0, \varphi, 0),\left(\Phi_{\mathcal{C}}\right)^{-1}(0,0, \psi)\right]\right)=(-[\varphi, \psi],[\varphi, \psi],[\varphi, \psi]) .
$$

It follows that $[\varphi, \psi] \in \operatorname{Coder}(\mathcal{C}) \cap{ }_{A} \operatorname{End}^{\mathcal{C}}(\mathcal{C})=0$ by Lemma $2.3(2)$.

Lemma 2.3 and Corollaries 3.3 and 5.2 immediately imply the next.

Corollary 5.3. The following hold.

(1) $\operatorname{GCoder}(\mathcal{C})$ and $\mathrm{SGCoder}(\mathcal{C})$ are Lie subalgebras of $\mathfrak{g l}(\mathcal{C})$.

(2) $\operatorname{GCoder}(\mathcal{C})$ is the semidirect product of the Lie subalgebra $\operatorname{Coder}(\mathcal{C})$ by the ideal ${ }_{A} \operatorname{End}^{\mathcal{C}}(\mathcal{C})$.

(3) $\operatorname{GCoder}(\mathcal{C})$ is the semidirect product of the Lie subalgebra $\operatorname{Coder}(\mathcal{C})$ by the ideal $^{\mathcal{C}} \operatorname{End}_{A}(\mathcal{C})$.

(4) SGCoder $(\mathcal{C})$ is the semidirect product of the Lie subalgebra $\operatorname{Coder}(\mathcal{C})$ by the ideal $^{\mathcal{C}} \operatorname{End}^{\mathcal{C}}(\mathcal{C})$.

Corollary 5.4. $\operatorname{GCoder}(\mathcal{C}) / \operatorname{GInCoder}(\mathcal{C}) \simeq \operatorname{Coder}(\mathcal{C}) / \operatorname{In} \operatorname{Coder}(\mathcal{C})$ as Lie algebras. 
Proof. By Corollary 5.2, $\operatorname{GInCoder}(\mathcal{C})$ is an ideal of $\operatorname{GCoder}(\mathcal{C})$. By Lemma 4.1 we get the assertion.

The next is an immediate consequence of Theorems 1.3 and 4.3 and Corollary 4.4.

Corollary 5.5. TInCoder $(\mathcal{C})$ is an ideal of the Lie algebra $\operatorname{TCoder}(\mathcal{C})$ and

$$
\operatorname{TCoder}(\mathcal{C}) / \operatorname{TInCoder}(\mathcal{C}) \simeq \operatorname{Coder}(\mathcal{C}) / \operatorname{InCoder}(\mathcal{C})
$$

as Lie algebras.

Theorem 5.6. Let $\mathcal{C}$ be an $A$-coring. Then $\operatorname{TCoder}(\mathcal{C})$ is a Lie subalgebra of the product Lie algebra $\mathrm{GCoder}(\mathcal{C})^{3}$, the $R$-module

$$
L=\operatorname{Coder}(\mathcal{C}) \times{ }_{A} \operatorname{End}^{\mathcal{C}}(\mathcal{C}) \times{ }^{\mathcal{C}} \operatorname{End}_{A}(\mathcal{C})
$$

is a Lie algebra by the Lie bracket

$$
\begin{aligned}
& {\left[(d, \varphi, \psi),\left(d^{\prime}, \varphi^{\prime}, \psi^{\prime}\right)\right]} \\
& =\left(\left[d, d^{\prime}\right],\left[d, \varphi^{\prime}\right]+\left[\varphi, d^{\prime}\right]+\left[\varphi, \varphi^{\prime}\right],\left[d, \psi^{\prime}\right]+\left[\psi, d^{\prime}\right]+\left[\psi, \psi^{\prime}\right]\right),
\end{aligned}
$$

and the map $\Phi_{\mathcal{C}}:$ TCoder $(\mathcal{C}) \rightarrow L$ given in Theorem 1.3 is a Lie algebra isomorphism.

Proof. By Lemma 5.1 and Corollaries 2.5 and 5.3, TCoder $(\mathcal{C})$ is a Lie subalgebra of GCoder $(\mathcal{C})^{3}$. Using Corollary 5.2 , it is easy to see that

$$
\left(\Phi_{\mathcal{C}}\right)^{-1}([x, y])=\left[\left(\Phi_{\mathcal{C}}\right)^{-1}(x),\left(\Phi_{\mathcal{C}}\right)^{-1}(y)\right] \quad(x, y \in L) .
$$

Hence $L$ is a Lie algebra and $\Phi_{\mathcal{C}}$ is a Lie algebra isomorphism.

In virtue of Theorem 5.6 we can define Lie algebra maps

$$
\begin{aligned}
& \lambda:{ }_{A} \operatorname{End}^{\mathcal{C}}(\mathcal{C}) \times{ }^{\mathcal{C}} \operatorname{End}_{A}(\mathcal{C}) \ni(\varphi, \psi) \mapsto(\varphi+\psi, \varphi, \psi) \in \operatorname{TCoder}(\mathcal{C}) \text { and } \\
& \mu: \operatorname{TCoder}(\mathcal{C}) \ni(f, g, h) \mapsto g+h-f \in \operatorname{Coder}(\mathcal{C}),
\end{aligned}
$$

where ${ }_{A} \operatorname{End}^{\mathcal{C}}(\mathcal{C}) \times{ }^{\mathcal{C}} \operatorname{End}_{A}(\mathcal{C})$ is the Lie subalgebra of $\mathfrak{g l}(\mathcal{C}) \times \mathfrak{g l}(\mathcal{C})$. It is easy to see that the Lie algebra $L$ defined in Theorem 5.6 is a semidirect product of the Lie subalgebra $\operatorname{Coder}(\mathcal{C}) \times 0 \times 0$ by the ideal $0 \times{ }_{A} \operatorname{End}^{\mathcal{C}}(\mathcal{C}) \times{ }^{\mathcal{C}} \operatorname{End}_{A}(\mathcal{C})$. Therefore we get the next.

Corollary 5.7. Under the above notations, the sequence of Lie algebra maps

$$
{ }_{A} \operatorname{End}^{\mathcal{C}}(\mathcal{C}) \times{ }^{\mathcal{C}} \operatorname{End}_{A}(\mathcal{C}) \stackrel{\lambda}{\longrightarrow} \operatorname{TCoder}(\mathcal{C}) \stackrel{\mu}{\longrightarrow} \operatorname{Coder}(\mathcal{C})
$$

is a split extension. 


\section{Triple derivations and triple coderivations}

Let $\mathcal{A}$ be an associative $R$-algebra with a unit 1 and $\mathcal{X} \in{ }_{\mathcal{A}} \mathbf{M}_{\mathcal{A}}$. A map $f$ in $\operatorname{Hom}_{R}(\mathcal{A}, \mathcal{X})$ is called a derivation if $f(\alpha \beta)=f(\alpha) \beta+\alpha f(\beta)$ for all $\alpha, \beta \in \mathcal{A}$. A map $f$ in $\operatorname{Hom}_{R}(\mathcal{A}, \mathcal{X})$ is called a generalized derivation if $f(\alpha \beta)=f(\alpha) \beta+$ $\alpha f(\beta)-\alpha f(1) \beta$ for all $\alpha, \beta \in \mathcal{A}$. A triplet $(f, g, h)$ in $\operatorname{Hom}_{R}(\mathcal{A}, \mathcal{X})^{3}$ is called a triple derivation if $f(\alpha \beta)=g(\alpha) \beta+\alpha h(\beta)$ for all $\alpha, \beta \in \mathcal{A}$. A generalized derivation was introduced in [1] and [8], and a triple derivation was introduced in [6] and studied in [5].

We define the $R$-linear map

$$
t_{\mathcal{X}}: \operatorname{Hom}_{R}(\mathcal{A}, \mathcal{X})^{3} \rightarrow \operatorname{Hom}_{R}\left(\mathcal{A} \otimes_{R} \mathcal{A}, \mathcal{X}\right)
$$

by setting $t_{\mathcal{X}}(f, g, h)(\alpha \otimes \beta)=f(\alpha \beta)-g(\alpha) \beta-\alpha h(\beta)$. Then

(1) $f$ is a derivation if and only if $t_{\mathcal{X}}(f, f, f)=0$.

(2) $f$ is a generalized derivation if and only if $t_{\mathcal{X}}(f, f, f) \in{ }_{\mathcal{A}} \operatorname{Hom}_{\mathcal{A}}\left(\mathcal{A} \otimes_{R} \mathcal{A}, \mathcal{X}\right)$.

(3) $(f, g, h)$ is a triple derivation if and only if $t_{\mathcal{X}}(f, g, h)=0$.

(1) and (3) are obvious. (2) follows from the fact that

$$
t_{\mathcal{X}}(f, f, f)(\alpha \otimes \beta)-\alpha t_{\mathcal{X}}(f, f, f)(1 \otimes 1) \beta=f(\alpha \beta)-f(\alpha) \beta-\alpha f(\beta)+\alpha f(1) \beta
$$

for all $\alpha, \beta \in \mathcal{A}$.

Let $\mathcal{C}$ be an $A$-coring and $B$ an $A$-ring, i.e., $B$ is an $R$-algebra with an $R$-algebra map $\eta: A \rightarrow B$ which maps a unit to a unit. Then $\mathcal{C}^{*}={ }_{A} \operatorname{Hom}_{A}(\mathcal{C}, B)$ is an $R$-algebra by the convolution product $\alpha * \beta=\mu \circ(\alpha \otimes \beta) \circ \Delta$ for $\alpha, \beta \in \mathcal{C}^{*}$ with the unit $\eta \circ \varepsilon$, where $\mu$ is the product of $B$. If $M \in{ }^{\mathcal{C}} \mathbf{M}^{\mathcal{C}}$, then $M^{*}={ }_{A} \operatorname{Hom}_{A}(M, B)$ is a right $\mathcal{C}^{*}$-module by the convolution product $\xi * \alpha=\mu \circ(\xi \otimes \alpha) \circ \rho^{M}$ for $\xi \in M^{*}$ and $\alpha \in \mathcal{C}^{*}$. Symmetrically $M^{*}$ has a left $\mathcal{C}^{*}$-module structure, and then $M^{*}$ is a $\left(\mathcal{C}^{*}, \mathcal{C}^{*}\right)$-bimodule. For $f \in{ }_{A} \operatorname{Hom}_{A}(M, \mathcal{C})$, we set $f^{*}={ }_{A} \operatorname{Hom}_{A}(f, B): \mathcal{C}^{*} \rightarrow M^{*}$. By definition, for any $f, g, h \in{ }_{A} \operatorname{Hom}_{A}(M, \mathcal{C})$, we can see that

$$
t_{M^{*}}\left(f^{*}, g^{*}, h^{*}\right)(\alpha \otimes \beta)=\mu \circ(\alpha \otimes \beta) \circ T_{M}(f, g, h) \quad\left(\alpha, \beta \in \mathcal{C}^{*}\right) .
$$

Therefore if $(f, g, h) \in \operatorname{TCoder}(M, \mathcal{C})$, then $\left(f^{*}, g^{*}, h^{*}\right)$ is a triple derivation. Furthermore using the equation (6.1), we can see that

$$
\begin{aligned}
& t_{M^{*}}\left(f^{*}, g^{*}, h^{*}\right)(\alpha \otimes \beta * \gamma)=\mu \circ\left(I_{B} \otimes \mu\right) \circ(\alpha \otimes \beta \otimes \gamma) \circ\left(I_{\mathcal{C}} \otimes \Delta\right) \circ T_{M}(f, g, h), \\
& t_{M^{*}}\left(f^{*}, g^{*}, h^{*}\right)(\alpha \otimes \beta) * \gamma=\mu \circ\left(\mu \otimes I_{B}\right) \circ(\alpha \otimes \beta \otimes \gamma) \circ\left(T_{M}(f, g, h) \otimes I_{\mathcal{C}}\right) \circ \rho^{M}
\end{aligned}
$$

for all $\alpha, \beta, \gamma \in \mathcal{C}^{*}$. Therefore if $T_{M}(f, g, h)$ is a right $\mathcal{C}$-comodule map, then $t_{M^{*}}\left(f^{*}, g^{*}, h^{*}\right)$ is a right $\mathcal{C}^{*}$-module map. Similarly if $T_{M}(f, g, h)$ is a left $\mathcal{C}$ comodule map, then $t_{M^{*}}\left(f^{*}, g^{*}, h^{*}\right)$ is a left $\mathcal{C}^{*}$-module map. 
Now suppose that $B$ is the tensor $A$-ring of the $(A, A)$-bimodule $\mathcal{C}$, i.e., $B=$ $A \oplus \bigoplus_{n=1}^{\infty} \underbrace{\mathcal{C} \otimes_{A} \cdots \otimes_{A} \mathcal{C}}_{n}$, and suppose that $\alpha, \beta$, and $\gamma$ are the canonical injection $\mathcal{C} \rightarrow B$. Then $\mu \circ(\alpha \otimes \beta)$ and $\mu \circ\left(\mu \otimes I_{B}\right) \circ(\alpha \otimes \beta \otimes \gamma)$ are monomorphisms. Therefore if $\left(f^{*}, g^{*}, h^{*}\right)$ is a triple derivation, then $(f, g, h) \in \operatorname{TCoder}(M, \mathcal{C})$, and if $t_{M^{*}}\left(f^{*}, g^{*}, h^{*}\right)$ is a $\left(\mathcal{C}^{*}, \mathcal{C}^{*}\right)$-bimodule map, then $T_{M}(f, g, h)$ is a $(\mathcal{C}, \mathcal{C})$-bicomodule map. Thus we get the next.

Theorem 6.1. Let $\mathcal{C}$ be an $A$-coring, $M \in{ }^{\mathcal{C}} \mathbf{M}^{\mathcal{C}}$, and $f, g, h \in{ }_{A} \operatorname{Hom}_{A}(M, \mathcal{C})$. Then the following hold.

(1) $f \in \operatorname{Coder}(M, \mathcal{C})$ if and only if for any $A$-ring $B,{ }_{A} \operatorname{Hom}_{A}(f, B)$ is a derivation.

(2) $f \in \operatorname{GCoder}(M, \mathcal{C})$ if and only if for any $A$-ring $B,{ }_{A} \operatorname{Hom}_{A}(f, B)$ is a generalized derivation.

(3) $(f, g, h) \in \operatorname{TCoder}(M, \mathcal{C})$ if and only if for any A-ring $B$, the triplet $\left({ }_{A} \operatorname{Hom}_{A}(f, B),{ }_{A} \operatorname{Hom}_{A}(g, B),{ }_{A} \operatorname{Hom}_{A}(h, B)\right)$ is a triple derivation.

Acknowledgment. The authors would like to express their gratitude to the referee for his valuable comments.

\section{References}

[1] M. Brešar, On the distance of the composition of two derivations to the generalized derivations, Glasgow Math. J., 33(1) (1991), 89-93.

[2] T. Brzeziński and R. Wisbauer, Corings and Comodules, Cambridge University Press, Cambridge, 2003.

[3] Y. Doi, Homological coalgebra, J. Math. Soc. Japan, 33(1) (1981), 31-50.

[4] F. Guzman, Cointegrations, relative cohomology for comodules, and coseparable corings, J. Algebra, 126(1) (1989), 211-224.

[5] H. Komatsu and A. Nakajima, Generalized derivations of associative algebras, Quaest. Math., 26(2) (2003), 213-235.

[6] G. F. Leger and E. M. Luks, Generalized derivations of Lie algebras, J. Algebra, 228(1) (2000), 165-203.

[7] A. Nakajima, Coseparable coalgebras and coextensions of coderivations, Math. J. Okayama Univ., 22(2) (1980), 145-149.

[8] A. Nakajima, On categorical properties of generalized derivations, Sci. Math., $2(3)$ (1999), 345-352.

[9] A. Nakajima, On generalized coderivations, Int. Electron. J. Algebra, 12 (2012), $37-52$. 


\section{Hiroaki Komatsu}

Faculty of Computer Science and Systems Engineering

Okayama Prefectural University

Soja, 719-1197, Japan

e-mail: komatsu@cse.oka-pu.ac.jp

\section{Atsushi Nakajima}

327-1, Nakaku, Nakai

Okayama, 703-8205, Japan

e-mail: a2017bj.naka@hi2.enjoy.ne.jp 